\title{
OP01
}

\section{FLICKER (PSTLM) AND STROBOSCOPIC EFFECT (SVM) - LIGHT MEASUREMENTS IN PHOTOMETRICAL LABORATORIES. SIGNIFY DEVELOPED SETUP AND VALIDATION METHOD}

\author{
Adam Klej et al.
}

DOI 10.25039/x46.2019.OP01

from

CIE x046:2019

\author{
Proceedings \\ of the \\ 29th CIE SESSION
}

Washington D.C., USA, June 14 - 22, 2019

(DOI $10.25039 / \times 46.2019)$

The paper has been presented at the 29th CIE Session, Washington D.C., USA, June 14-22, 2019. It has not been peer-reviewed by CIE.

(C) CIE 2019

All rights reserved. Unless otherwise specified, no part of this publication may be reproduced or utilized in any form or by any means, electronic or mechanical, including photocopying and microfilm, without permission in writing from CIE Central Bureau at the address below. Any mention of organizations or products does not imply endorsement by the CIE.

This paper is made available open access for individual use. However, in all other cases all rights are reserved unless explicit permission is sought from and given by the CIE.

CIE Central Bureau

Babenbergerstrasse 9

A-1010 Vienna

Austria

Tel.: +4317143187

e-mail: ciecb@cie.co.at

www.cie.co.at 


\title{
FLICKER (PST ${ }^{\text {LM }}$ ) AND STROBOSCOPIC EFFECT (SVM) - LIGHT MEASUREMENTS IN PHOTOMETRICAL LABORATORIES. SIGNIFY DEVELOPED SETUP AND VALIDATION METHOD
}

\author{
Adam Klej ${ }^{1}$, Andrew Jackson ${ }^{2}$, Pierre Beeckman ${ }^{1}$, Norbert Mila ${ }^{3}$, Konika Banerjee ${ }^{1}$, Marc \\ Embrechts ${ }^{4}$, Henk-Jan van Aalderen ${ }^{1}$ \\ ${ }^{1}$ Signify, Eindhoven, NETHERLANDS, ${ }^{2}$ Signify, Salina, USA, ${ }^{3}$ Signify, Shanghai, CHINA, \\ ${ }^{4}$ Signify, Turnhout, BELGIUM \\ adam.klej@signify.com
}

DOI $10.25039 / \times 46.2019 .0 P 01$

\begin{abstract}

\section{Motivation, specific objective}

The document CIE TN 006:2016 - Visual Aspects of Time-Modulated Lighting Systems Definitions and Measurement Models contains the description of current measures to evaluate Temporal Light Artifacts (TLA), including flicker and stroboscopic effects. From an industry perspective, there are several methods available to record optical waveforms and to evaluate these waveforms for TLA effects. These TLA metrics can be utilized to judge the quality of lighting products. The primary documents that are currently available for test and measurement of optical waveforms and TLA are: [1] National Electrical Manufacturers Association (NEMA) NEMA 77-2017 Temporal Light Artifacts: Test Methods and Guidance; [2] International Electrotechnical Commission (IEC) TR 61547-1:2017 ED2 Equipment for general lighting purposes - EMC immunity requirements - Part 1: An objective light flickermeter and voltage fluctuation immunity test method; and [3] (IEC) TR 63158:2018 Equipment for general lighting purposes - Objective test method for stroboscopic effects of lighting equipment. These three documents provide guidance for performing accurate measurements, but many laboratories struggle making consistent and accurate measurements of temporal light artefacts.
\end{abstract}

Keywords: Photometry, TLA, stroboscopic effect, flicker effect, $\mathrm{P}_{\mathrm{st}}{ }^{\mathrm{LM}}$, SVM, TLM

\section{Methods}

Signify developed a measurement setup and verification methods for TLA, utilizing a novel reference modulation radiator (aka Reference Radiator) to validate the TLA measurement systems.

\subsection{TLA Acquisition setup}

The test set-up utilized in Signify follow the approach outlined in NEMA-77 and in the IEC documents and $[1,2,3]$ and a block-diagram of the setup is presented in Figure 1.

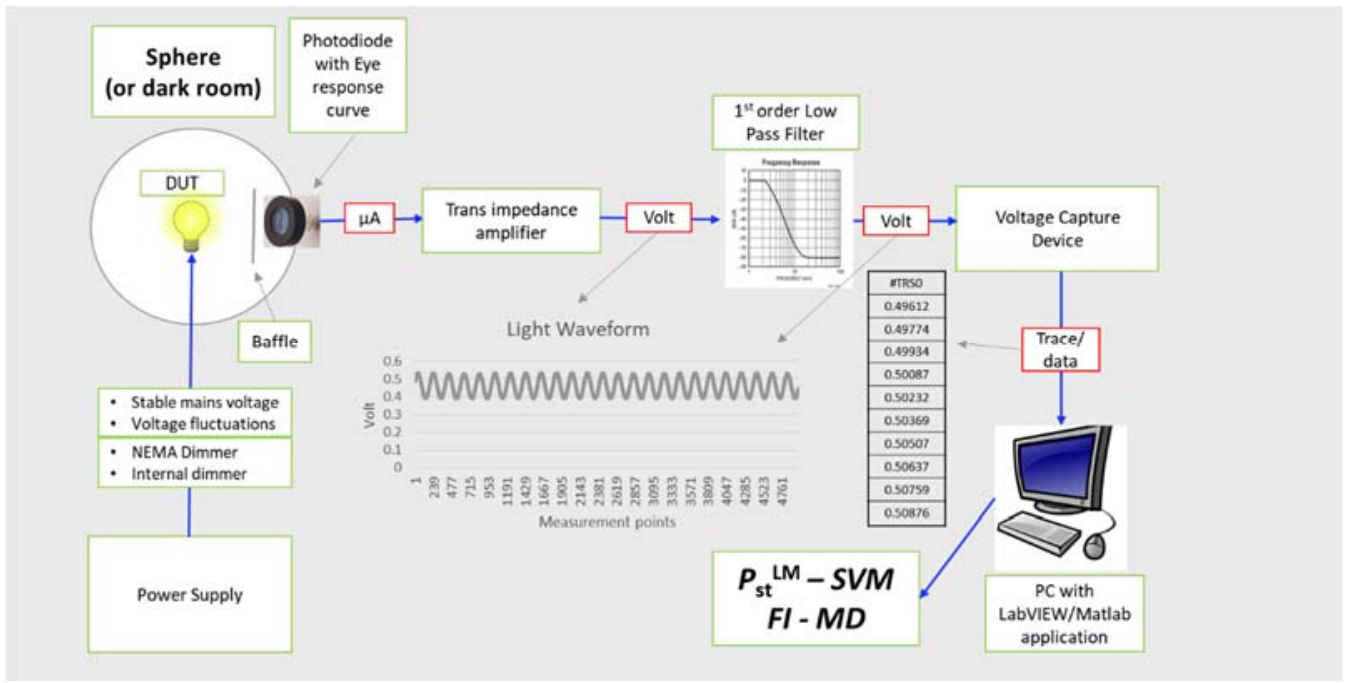

Figure 1 - Signify TLA Acquisition setup scheme 
The measurement Setup contains:

- Power Supply

- Photodiode with Eye response curve

- Trans-Impendence Amplifier

- $1^{\text {ST }}$ Order Low Pass filter

- Voltage Capture Device

- Acquisition Software developed by Signify

\subsection{Signify TLA Reference Radiator}

Proper verification of the TLA setup can be challenging due to novelty of measurement methods and often not knowing the limitations of TLA acquisition setups that may cause wrong measurements of TLA. The Signify TLA Reference Radiator (Figure 2) can be used to evaluate the measurement abilities of the setup. The radiator generates pre-programmed periodic light waveforms with different shapes, modulation frequencies \& modulation levels. The exact values of the TLA metrics ( $P_{s t}{ }^{L M}$, SVM, FI, MD) are known for the input waveforms (mathematical representations) and it can generate very stable and reproducible optical signals. The unit is used to:

- Demonstrate TLA effects (education)

- Examine the appropriateness of existing metrics

- Validate TLA test set-ups

- Provide a controlled round robin sample for interlaboratory comparison - there are more than 26 optical waveshapes that have been programmed into the unit

- And may be used to test metric limits (research)
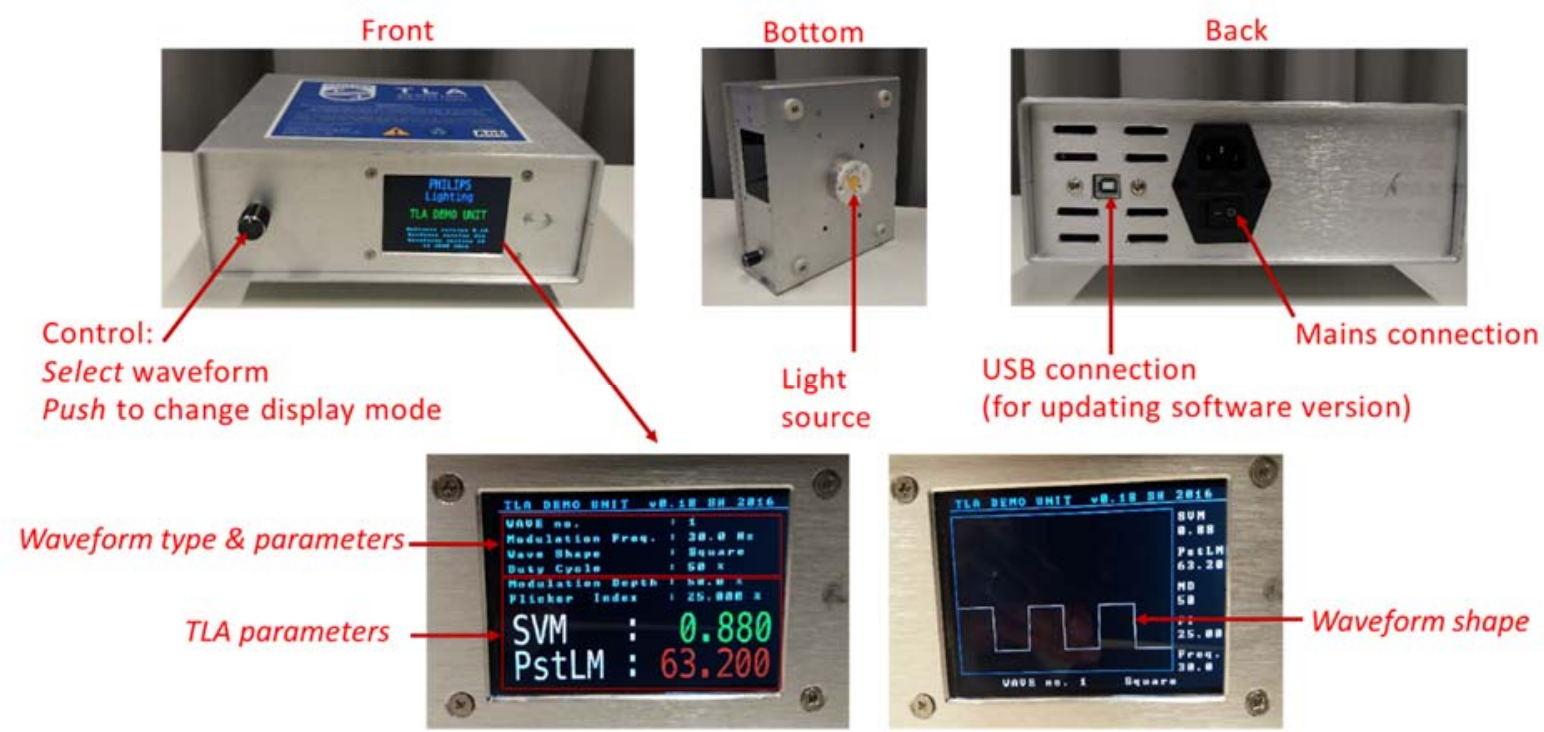

Figure 2 - Signify Reference Radiator - Description

Figure 3 shows example Ideal vs. measured sawtooth waveform \#9 which has a modulation depth of $100 \%$ and a frequency of $50 \mathrm{~Hz}$. The graph shows a good linear relationship between applied LED current waveform and actual measured light waveform. 


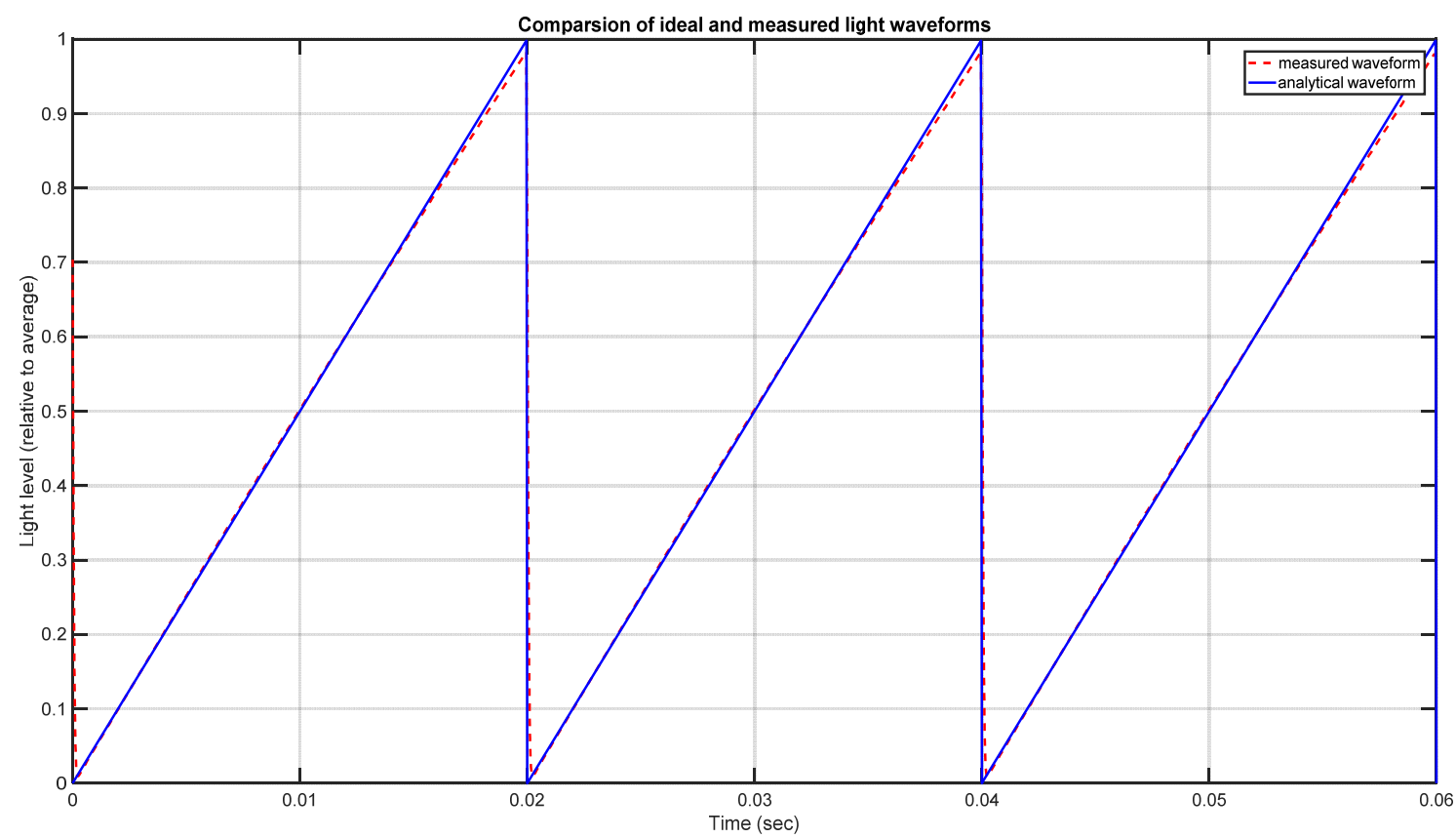

Figure 3 - Example waveform \#9 analytical (ideal) and measured waveforms

\section{Results}

For the verification of the Reference Radiator, the mathematically calculated values were compared with the measured values. Graph (Figure 4) shows a good correlation for $\mathrm{P}_{\mathrm{st}}{ }^{\mathrm{LM}}$ and SVM between measured and numerical/analytical values of the waveforms.
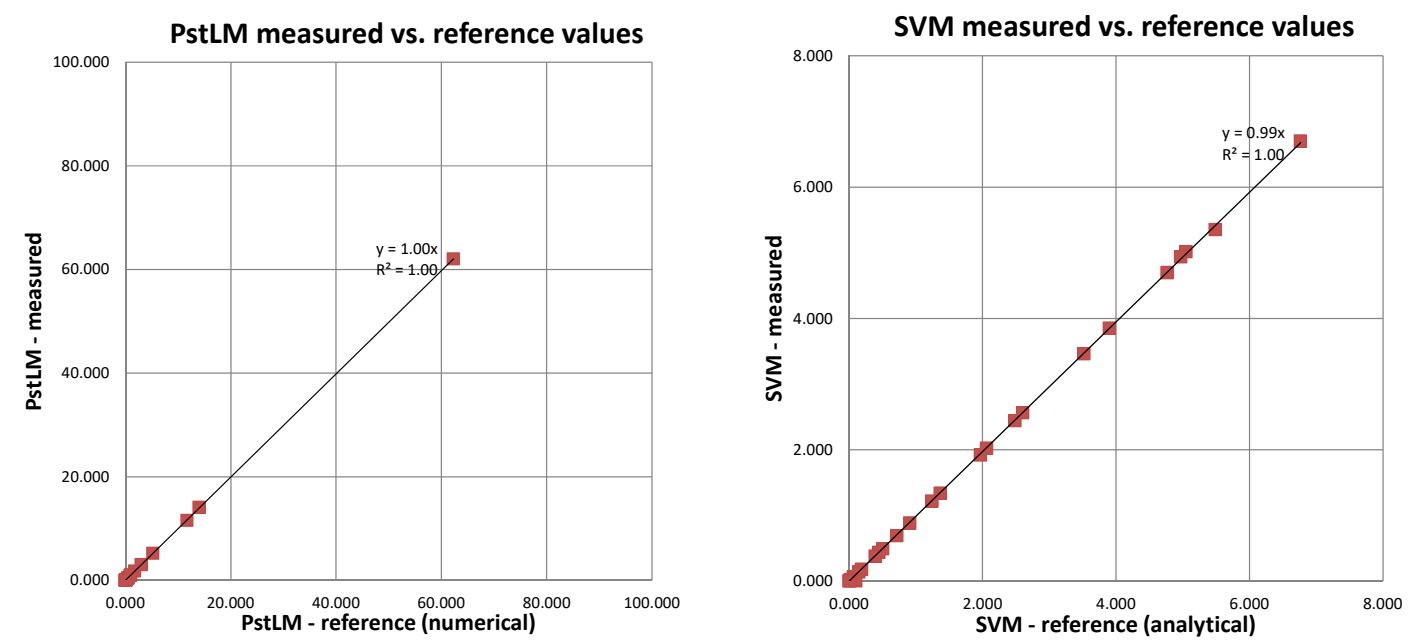

Figure $4-P_{s t}{ }^{L M}$ and SVM measured values vs reference values

Additionally, the reference radiator was utilized to carry out an interlaboratory comparison between three light measurement laboratories. Several pre-programmed waveforms of which the TLA metrics were known analytically were chosen for the comparison. The TLA measurement capabilities as well as the limitations of the laboratories were examined. The proper acquisition of the light waveforms and the proper data processing and calculation of the metrics were the main points of attention of this study. 


\section{Conclusions}

A proper verification of the TLA measurement system and, knowing its limitation is important to ensure high quality measurements at Photometrical Laboratories. The verification method developed by Signify using the reference radiator provides a solution to laboratories to have the ability to measure and calculate quantities correctly. Interlaboratory comparison results show alignment between laboratories where the reference radiator was utilized for verification of the set-up. The first two NVLAP accredited laboratories to the NEMA 77-2017 standard were Signify Laboratories - Salina/USA and Shanghai/China. This proves that Signify verification method helps reach ability and reproducibility of measured quantities at the developed setups. Signify experts are also involved in Division 2 TC2-89 activities on TLA/TLM Measurements Technical Note, which is guidance document for Photometrical Laboratories to enable proper measurements. More details on Reference Radiator abilities and results of comparison of results between Signify Lighting Test Centers will be presented during the oral presentation at CIE conference.

\section{References}

[1] National Electrical Manufacturers Association (NEMA) NEMA 77-2017 Temporal Light Artifacts: Test Methods and Guidance;

[2] International Electrotechnical Commission (IEC) TR 61547-1:2017 ED2 Equipment for general lighting purposes - EMC immunity requirements - Part 1: An objective light flickermeter and voltage fluctuation immunity test method; and

[3] International Electrotechnical Commission (IEC) TR 63158:2018 Equipment for general lighting purposes - Objective test method for stroboscopic effects of lighting equipment. 